Investigación

\title{
CONOCIMIENTO Y SOBRECARGA DEL CUIDADOR PRINCIPAL DE PERSONA MAYOR DEPENDIENTE
}

\author{
KNOWLEDGE AND BURDEN OF THE MAIN CAREGIVER OF A DEPENDENT \\ ELDERLY PERSON
}

\author{
Graciela Olarte Rueda \\ Enfermera \\ (C) Mg en ciencia de la enfermería, Especialista en epidemiología. Facultad Ciencias de la \\ Educación y de la Salud, UNISANGIL. San Gil, Colombia \\ grueda@unisangil.edu.co \\ https://orcid.org/0000-0003-1733-3285

\section{Yineth Cristina Largo Cardenas}

\section{Enfermera} \\ Facultad Ciencias de la Educación y de la Salud, UNISANGIL. San Gil, Colombia \\ Yinethlargo@unisangil.edu.co \\ https://orcid.org/0000-0001-6664-3242
}

\section{Deisy Lizeth León Acosta}

Enfermera

Facultad Ciencias de la Educación y de la Salud, UNISANGIL. San Gil, Colombia deiyleon@unisangil.edu.co

https://orcid.org/0000-0003-0932-6640

\section{Linda Sarid Del Rosio Sánchez Cubides}

\section{Enfermera}

Facultad Ciencias de la Educación y de la Salud, UNISANGIL. San Gil, Colombia lindasanchez@unisangil.edu.co

https://orcid.org/0000-0002-3861-6666

\section{Liliana Marcela Ríos Gómez}

\section{Enfermera}

Facultad Ciencias de la Educación y de la Salud, UNISANGIL. San Gil, Colombia lilianarios@unisangil.edu.co

https://orcid.org/0000-0002-3861-6666

$\overline{\text { Artículo recibido el } 21 \text { de octubre de 2021. Aceptado en versión corregida el } 6 \text { de diciembre }}$ de 2021. 


\title{
RESUMEN
}

INTRODUCCIÓN: Los cuidadores son los encargados de brindar apoyo a las necesidades básicas de persona mayor. OBJETIVO: Determinar los conocimientos y sobrecarga en el cuidador principal de persona mayor dependiente del municipio de San Gil Santander. MATERIALES Y MÉTODOS: el estudio fue de tipo descriptivo, transversal, la muestra estuvo conformada por 50 cuidadores informales, la recolección de datos se realizó mediante los instrumentos de antecedentes demográficos, evaluación del conocimiento, índice de Katz para medir el grado de dependencia en el adulto mayor y la escala de Zarit para analizar el nivel de sobrecarga en el cuidador. RESULTADOS: El nivel de conocimiento de los cuidadores principales de persona mayor se encontró que el $66 \%$ conocen los beneficios de la alimentación, el $68 \%$ tienen una incapacidad severa y los cuidadores presentan una sobrecarga leve en 34\%. CONCLUSIÓN: los cuidadores presentaron un déficit de conocimientos en técnicas de mecánica corporal, técnicas para manejo del estrés y en la importancia de la utilización de los elementos de protección personal, por otra parte, a los adultos mayores tenían una dependencia severa y los cuidadores presentaron una sobrecarga leve.

Palabras clave: Cuidador, cuidado, persona, conocimiento, sobrecarga (DECS).

\begin{abstract}
INTRODUCTION: Caregivers are in charge of providing support for the basic needs of the elderly. OBJECTIVE: To determine the knowledge and overload in the main caregiver of a dependent elderly person in the municipality of San Gil Santander. MATERIALS AND METHODS: the study was descriptive, cross-sectional, the sample was made up of 50 informal caregivers, data collection was carried out using the instruments of demographic background, knowledge evaluation, Katz index to measure the degree of dependence on the older adult and the Zarit scale to analyze the level of burden in the caregiver. Results: The level of knowledge of the main caregivers of the elderly, it was found that $66 \%$ know the benefits of feeding, $68 \%$ have a severe disability and the caregivers present a slight overload in 34\%. CONCLUSION: the caregivers presented a knowledge deficit in body mechanics techniques, stress management techniques and the importance of the use of personal protection elements, on the other hand, older adults had a severe dependency and the caregivers presented slight overload.
\end{abstract}

Key words: Caregiver, care, person, knowledge, overload (DECS)

http://dx.doi.org/10.7764/Horiz_Enferm.32.3.256-265

\section{INTRODUCCIÓN}

El cuidador principal es considerado como aquella persona que se hace cargo de manera permanente de las necesidades

2021. HorizEnferm, 32,3,256-265 básicas de la persona mayor; generalmente este resulta ser un familiar o persona cercana $^{(1)}$, siendo esta actividad conside- 
Conocimiento y sobrecarga del cuidador principal de persona mayor dependiente

rada un riesgo debido a que pueden presentar una sobrecarga manifestada por agotamiento, ansiedad y sentimientos negativos; muchos de esos síntomas están relacionados con que llevan más de 5 años en esta Situación laboral ${ }^{(2)}$, con horarios mayor a 12 horas diarias, por lo tanto, se hace necesario evaluar el impacto que su rol de cuidador que tiene en su salud ${ }^{(3)}$.

Teniendo en cuenta que la dependencia es un estado de carácter permanente en que se encuentran las personas por razones derivadas de la edad, la enfermedad o la discapacidad, ligadas a la falta o a la pérdida de autonomía física, mental, intelectual o sensorial que precisan de la atención de otra persona o ayudas importantes para realizar actividades básicas de la vida diaria ${ }^{(4)}$.

El grado de dependencia se clasifica en grado I dependencia moderada en el grado II la dependencia severa y en el grado III gran dependencia por esta razón requiere apoyo indispensable y continuo de otra persona ${ }^{(5)}$.

El cuidado de la persona mayor representa una serie de actos de vida que tienen por finalidad y función mantener y perpetuar la $\mathrm{vida}^{(6)}$, de tal forma el cuidador principal es la persona que dedica la mayor parte de su tiempo al cuidado, es aquel que asume las principales tareas y responsabilidades ${ }^{(7)}$, las cuales se pueden ver afectadas por diversos factores como la edad y falta de apoyo familiar, además pueden presentar problemas osteomusculares debido a los esfuerzos realizados; provocando alteraciones en el desempeño de su labor, afectando su propia satisfacción personal ${ }^{(8)}$.

Por lo anterior, fue fundamental indagar sobre las habilidades que poseían los cuidadores principales y de esta forma diseñar intervenciones que mejoren la calidad de la atención y se disminuya la sobrecarga $^{(9)}$, asimismo, es importante que se dé un reconocimiento en el desempeño del rol cuidador puesto que esta labor tiene una gran relevancia personal, familiar y social que en el momento no se le ha dado la importancia que merece ${ }^{(10)}$.

Fue importante desarrollar esta investigación ya que sirvió de base para implementar y desarrollar futuras investígaciones e intervenciones que puedan mitigar este problema social que afecta a las personas cuidadoras del adulto mayor, en su mayoría a las mujeres que son las principales proveedoras de cuidado ${ }^{(11)}$, las cuales se ven afectadas debido a que tienen múltiples responsabilidades en su hogar y vida personal, donde por falta de apoyo esto se convierte en una sobrecarga haciendo que su desempeño disminuya en efectividad y calidad, generando agotamiento y abandono de su labor ${ }^{(12)}$.

Por lo anterior el objetivo general del estudio fue determinar los conocimiento y sobrecarga en el cuidador principal de persona mayor dependiente en el municipio de San Gil Santander, y como objetivos específicos: caracterizar socio demográficamente a los cuidadores de la persona mayor dependiente en el municipio de San Gil Santander; medir la sobrecarga de los cuidadores y las repercusiones que este rol representa e identificar el grado de dependencia de las personas mayores.

\section{MATERIALES Y MÉTODOS}

El estudio fue de tipo descriptivo de corte trasversal, para determinar los conocímientos que tiene los cuidadores en cuanto 
al cuidado, de igual forma describir el nivel de sobrecarga. Mediante muestreo a conveniencia se incluyó una muestra de 50 cuidadores principales de persona mayor dependiente en sus domicilios del municipio de San Gil Santander, que cumplieran con los criterios de inclusión cuidador principal mayor de 18 años, tiempo como cuidador mayor a 6 meses, que acepte la participación en el estudio y firme el consentimiento informado $y$ cuidadores principales que no presenten alteraciones a nivel mental o cognitivas que nos les permitan responder las preguntas realizadas en el estudio.

La recolección de los datos se realizó mediante el instrumento características sociodemográficas de los cuidadores informales el cual consta de seis preguntas: sexo, edad, nivel de educación, percepción de la situación económica, situación laboral actual y tiempo como cuidador; además se aplicó el instrumento Índice de $\operatorname{KATZ}^{(13)}$, cuestionario de 6 ítems, en los cuales se valoran las actividades de la vida diaria y la ayuda que requiere el adulto mayor para realizarlas con el que se determinó el grado de dependencia; igualmente se realizó la medición de la sobrecarga en los cuidadores mediante la escala de Zarit que consta de 22 ítems con respuesta tipo escala likert (1-5) ${ }^{(14)}$. Asimismo, se evaluó en nivel de conocimientos con un cuestionario de 10 preguntas diseñado exprofeso para este trabajo ${ }^{(15)}$.

El procesamiento y análisis estadístico se realizó a través del programa estadístico SPSS versión 24. En primer lugar, se hizo la descripción de las variables sociodemográficos de los cuidadores principales del adulto mayor incluidos en la base de datos mediante el análisis univariado. Las variables cualitativas son presentadas en términos de porcentajes y frecuencias, mientras que las variables cuantitativas se presentan en términos de medias, medianas, y desviación estándar.

\section{Consideración ética}

La investigación se consideró sin riesgo según la resolución 8430 de 1993, aprobado por el comité de ética institucional de Unisangil.

\section{RESULTADOS}

\section{Características sociodemográficas}

Se encontró que el $88 \% \quad(n=44)$ de los cuidadores principales son de género femenino, con promedio de edad de 52,6 años con un rango de edad de 18 a 83 años, desviación estándar $(15,948)$, mediana de (52,50), moda (50); se evidenció que el $48 \% \quad(n=24)$ de los cuidadores tienen estudios de primaria, además el $62 \%$ $(n=31)$ tienen una percepción económica regular, donde el 54\% $(\mathrm{n}=27)$ son amas de casa y el $48 \%(n=24)$ llevan de 1 a 5 años de tiempo en esta labor. Tabla 1. 
Conocimiento y sobrecarga del cuidador principal de persona mayor dependiente

Tabla 1. Aspectos sociodemográficos.

\begin{tabular}{|c|c|c|}
\hline Variable & Frecuencia & Porcentaje \\
\hline \multicolumn{3}{|l|}{ Género } \\
\hline Femenino & 44 & 88 \\
\hline Masculino & 6 & 12 \\
\hline Total & 50 & 100 \\
\hline \multicolumn{3}{|l|}{ Escolaridad } \\
\hline Sin estudio & 4 & 8,0 \\
\hline Primaria & 24 & 48,0 \\
\hline Secundaria & 14 & 28,0 \\
\hline Pregrado & 8 & 16,0 \\
\hline Total & 50 & 100,0 \\
\hline \multicolumn{3}{|l|}{ Situación Económica } \\
\hline Buena & 16 & \\
\hline Mala & 3 & 32,0 \\
\hline Regular & 31 & $\begin{array}{c}6,0 \\
62,0\end{array}$ \\
\hline Total & 50 & 100,0 \\
\hline \multicolumn{3}{|l|}{ Ocupación } \\
\hline Ama de casa & 27 & 54,0 \\
\hline Estudiante & 1 & \\
\hline Jubilado- Pensionado & 2 & 2,0 \\
\hline Trabajado tiempo & 13 & \\
\hline parcial & 7 & 4,0 \\
\hline $\begin{array}{l}\text { Trabajado tiempo } \\
\text { completo }\end{array}$ & & 26,0 \\
\hline & 50 & \\
\hline \multirow[t]{2}{*}{ Total } & & 14,0 \\
\hline & & 100 \\
\hline Tiempo & & \\
\hline Cuidador & 7 & \\
\hline Menos de un Año & 24 & 14,0 \\
\hline 1 a 5 años & 6 & 48,0 \\
\hline 5 a 10 años & 13 & 12,0 \\
\hline Más de 10 años & 50 & 26,0 \\
\hline Total & & 100,0 \\
\hline
\end{tabular}

Fuente: Autor. 
Olarte Rueda G, Largo Cardenas YC, León Acosta DL, Sánchez Cubides LSDR, Ríos

Gómez LM

\section{Evaluación del conocimiento para cuidadores informales}

En cuanto al nivel de conocimiento de los cuidadores principales de persona mayor se encontró que el $66 \%(n=33)$ conocen los beneficios de la alimentación que consume el adulto mayor y el 50\% $(n=50)$ utilizan la posición adecuada para dar la alimentación.

Por otra parte, el $72 \%(n=36)$ de los cuidadores principales utilizan su propia fuerza para los cambios de posición, el $64 \%(n=32)$ conocen los daños que se le pueden ocasionar a la persona mayor si no se le realiza ningún cambio de posición, de igual modo se demostró que $80 \% \quad(n=40)$ de los cuidadores principales no tienen conocimientos en técnicas de mecánica corporal y el $78 \%(n=39)$ no usan técnicas para manejo del estrés.

Finalmente, el $48 \% \quad(n=24)$ no utilizaron elementos de protección personal, también se encontró que el $62 \%$ $(n=31)$ no conocían el grado de dependencia que tiene la persona mayor, así mismo se pudo determinar que el $66 \%$ $(n=33)$ de los cuidadores llevan un listado de los medicamentos que necesita la persona mayor, de igual forma el $64 \%$ $(n=32)$ recibieron apoyo por parte de algún familiar.

\section{Nivel de dependencia}

Se encontró que el 68\% $(n=34)$ de las personas mayores tiene una incapacidad severa en el uso del baño, en el vestido, en la movilidad, en la continencia, la alimentación y uso del inodoro. Tabla 2.

Tabla 2. Grado de dependencia.

Frecuencia Porcentaje

\begin{tabular}{lcc}
\hline $\begin{array}{l}\text { Incapacidad } \\
\text { Moderada }\end{array}$ & 12 & 24,0 \\
$\begin{array}{l}\text { Incapacidad } \\
\text { Severa }\end{array}$ & 34 & 68,0 \\
Total & 50 & 100,0
\end{tabular}

Fuente: Índice de Katz.

\section{Sobrecarga del cuidador}

El 32\% ( $n=16)$ presentaron una sobrecarga intensa porque no reciben ayuda por parte de nadie para el cuidado y además los adultos mayores que cuidan son dependientes. Tabla 3.

Tabla 3. Nivel de sobrecarga.

\begin{tabular}{lcc}
\hline & Frecuencia & Porcentaje \\
\hline No Sobrecarga & 17 & 34,0 \\
Sobrecarga Intensa & 16 & 32,0 \\
Sobrecarga Leve & 17 & 34,0 \\
Total & 50 & 100,0 \\
\hline
\end{tabular}

Fuente: Escala de Zarit.

\section{DISCUSIÓN}

El propósito de esta investigación es determinar los conocimientos y sobrecarga en el cuidador principal de la persona mayor dependiente, para que así se pueda reconocer su labor y conocer sus necesidades frente al cuidado con el fin de alcanzar un alto nivel de calidad de vida al cuidador. 
Conocimiento y sobrecarga del cuidador principal de persona mayor dependiente

En esta investigación se encontró que el género predominante es el femenino esto coincide con el estudio de Silva, J. ${ }^{(16)}$, Humbelina A. ${ }^{(17)}$, Osorio, V. ${ }^{(18)}$ y Carreño, S. ${ }^{(19)}$. Lo que demuestra que en su mayoría los cuidadores de los adultos mayores son mujeres.

Teniendo en cuenta el perfil socio demográfico corresponde a mujeres mayores de 50 años en comparación al estudio realizado por De Valle et al. ${ }^{(20)}$, en el cual señala que las cuidadoras eran "mujeres mayores de 40 años"; por otro lado, en este estudio ellas poseen un nivel académico primaria y ocupación amas de casa así mismo en la investigación de Arbuès, Martínez y Gómez ${ }^{(21)}$, la hija de la persona tiene grados académicos de básico y primaria.

Por otro lado, mediante la evaluación de conocimientos la mayoría de los cuidadores principales no tiene conocimientos sobre mecánica corporal y técnica manejo del estrés, por lo tanto es fundamental que los cuidadores reciban instrucciones para brindar una mejor atención en salud del adulto(22), aunque que reciben apoyo por parte de algún familiar a diferencia en el estudio de Borda, J. ${ }^{(23)}$, donde el adulto mayor no acepta el cuidado de otro familiar diferente al que lo ha estado cuidando por un tiempo prologado.

De acuerdo a nivel de sobrecarga de los cuidadores en el estudio se encontró un porcentaje de $34 \%$ una sobrecarga leve, asimismo, en el estudio de Navarro et al. ${ }^{(24)}$, donde el $44,7 \%$ presentaban sobrecarga ligera, a diferencia del estudio de Rodriguez y Quemba ${ }^{(25)}$ "el nivel de carga presente fue de sobrecarga intensa $(35,7 \%)$ y sobrecarga leve (14,29\%)", asimismo, en el estudio de Piñeiro et al. ${ }^{(26)}$, encontraron un $30 \%$ en "sobrecarga intensa" $y$, finalmente, "sobrecarga leve" con un 10\% de los casos.

Asimismo, se evidenció que los cuidadores no utilizan, ni tiene conocimiento de la importancia de la utilización de los elementos de protección personal para el cuidado en relación con la investigación de Romero, Z. ${ }^{(27)}$ y Lemus, et al. (28) "los cuidadores no habían recibido capacitación para el cuidado". Esto es importante para que los cuidadores proporcionen técnicas adecuadas en caminadas a mantener una alta calidad de cuidado reflejada en un trato humanizado, respetando la vida y la integridad del anciano así mismo para que los cuidadores tengan un estado de salud óptimo minimizando las molestias ocasionadas por dicha labor, lo que implica que se realice un diagnóstico de las necesidades de los cuidadores y se desarrollen líneas de apoyo que puedan mejorar la situación de salud de los involucrados ${ }^{(29)}$.

\section{CONFLICTO DE INTERESES}

Las autoras declaran no tener ningún conflicto de interés.

\section{REFERENCIAS BIBLIOGRÁFICAS}

1. González, Emy; Orozco, Gladys; Poveda E a. Sobrecarga del Cuidador Versus Autonomía Funcional del Anciano. [Internet]. 2012 [citado 2020 Agosto 21] $14 \quad$ (8), 351-354. Disponible: http://riuc.bc.uc.edu.ve/bitstream/1234 56789/1983/1/art01.pdf

2. Martínez-aguilera P. Sobrecarga y Burnout en cuidadores informales del adulto mayor. Enferm Univ [Internet]. 
Olarte Rueda G, Largo Cardenas YC, León Acosta DL, Sánchez Cubides LSDR, Ríos

Gómez LM

2015; [citado 2019 Agosto 24] 12(1):19-27. Disponible : http://www.scielo.org.mx/pdf/eu/v12n 1/v12n1a4.pdf

3. Silva Fhon R , Gonzales Janampa T, Marques S, Partezani Rodrigues R, Mas Huaman T. Sobrecarga y calidad de vida del cuidador principal del adulto mayor Sobrecarga e qualidade de vida do cuidador principal do idoso Overburden and quality of life of the primary elderly caregiver. 2017; [citado 2019 septiembre 5] 251-8. Disponible: http://www.scielo.org.co/pdf/aven/v34 n3/v34n3a05.pdf

4. Retamal-matus H, Aguilar L, Aguilar M, González R, Valenzano S. Factores que infl uyen en la sobrecarga de los cuidadores de adultos mayores en dos instituciones de la ciudad de Punta Arenas , Chile. Dep Kinesiol Fac Ciencias la Salud Univ Magallanes Punta Arenas, Chile [Internet]. 2015; [citado Junio 2020] 5(3):119-24. Disponible:

https://www.viguera.com/sepg/pdf/revi sta/0503/503_0119_0124.pdf

5. Campos Dompedro JR, de Dios Estrella Cazalla J. Guía practica de la ley de dependencia. Soc Española Geriatría y Gerontol [Internet]. [citado abr de 2020].;1. Disponible: https://www.segg.es/media/descargas/ Guia práctica de la Ley de Dependencia.pdf

6. Ramírez Perdomo C, Parra Vargas M. Percepción de los comportamientos del cuidado de enfermería en la unidad de cuidados intensivos. Av en Enfermería [Internet] [citado 2019 julio 18] 1:97$108 . \quad$ Disponible: https://revistas.unal.edu.co/index.php/a venferm/article/view/35861/37067

7. Gómez-Martinho MR. Cuidado formal e informal de personas mayores dependientes. Univ Pontif ICAI ICADE Comillas Madrid [Internet]. 2016; [citado 2019 Septiembre 10] 1:134.

Disponible: https://repositorio.comillas.edu/xmlui/ bitstream/handle/11531/13437/TFM00 0507.pdf? sequence $=1$

8. Gómez Poyato J. Los cuidadores informales y su calidad de vida. Univ Zaragoza [Internet]. [citado 2019 Agosto 21] 1:13-21. Disponible: http://invenio2.unizar.es/record/11127/ files/TAZ-TFM-2013-221.pdf

9. Criado L, Vega OM, Cuenca I. Magnitud de efecto de un programa dirigido a cuidadores familiares de personas con enfermedad crónica. 2017; [citado Octubre 2020] 1:11-20. Disponible:

http://www.scielo.org.co/pdf/nova/v16 n29/1794-2470-nova-16-29-00011.pdf

10. Vega-angarita OM. Percepción del apoyo social funcional en cuidadores familiares de enfermos crónicos. [internet]. [citado Agosto 2019] 18 11:274-86. Disponible: http://www.scielo.org.co/pdf/aqui/v11 n3/v11n3a04.pdf

11. Humbelina Díaz A, Lemus Fajardo N, Gonzáles Cosme W, Licort Monduy O, Gort Cuba O. Repercusión ética del cuidador agotado en la calidad de vida de los ancianos. Rev Ciencias Médicas Pinar del Río [Internet]. 2015; [citado 2019 septiembre 8] 19(3). Disponible: http://scielo.sld.cu/scielo.php?script=s ci_arttext\&pid=S156131942015000300011

12. García MCP, Domínguez MLR. 
Conocimiento y sobrecarga del cuidador principal de persona mayor dependiente

Sobrecarga en cuidadores principales de Adultos Mayores. Rev Salud Pública Parag [Internet]. 2016; [citado 2019 mayo 16] 6:10-5. Disponible: http://docs.bvsalud.org/biblioref/2018/ 06/905082/10-15.pdf

13. Salud SA de. Valoración de las actividades de la vida diaria -índice de KATZ. Cons salud [Internet]. [citado 2019 mayo 12] :0-1. Disponible: http://www.hvn.es/enfermeria/ficheros /indice_de_katz.pdf

14. Servicios Andaluz de Salud, Consejería de Salud (s.f). Índice de KATZ. Recuperado de: http://www.hvn.es/enfermeria/ficheros /indice_de_katz.pdf

15. Burgos Garrido P,Figueroa Rodríguez V, Fuentes Verdugo M, Quijada Hernández I. nivel de conocimiento del cuidador informal de usuario con dependencia severa adscrito al consultorio violeta parra - CHILLÁN. Univ del BIO - BIO Fac ciencias la salud y los aliment esc enfermería. 2008; [citado 2019 julio 7] 1:1-82. Disponible:

http://repobib.ubiobio.cl/jspui/bitstrea m/123456789/783/1/Burgos

Garrido\%2C Paola Andrea.pdf

16. Silva Jack. Sobrecarga y calidad de vida del cuidador principal del adulto mayor. Artic Investig . 2017; [citado 2019 julio 28] 1:251-9. Dsiponible: http://www.scielo.org.co/pdf/aven/v34 n3/v34n3a05.pdf

17. Humbelina Díaz A, Lemus Fajardo N, Gonzáles Cosme W, Licort Monduy O, Gort Cuba O. Repercusión ética del cuidador agotado en la calidad de vida de los ancianos. Rev Ciencias Médicas Pinar del Río [Internet]. 2015; [citado
2019 septiembre 8] 19(3). Disponible: http://scielo.sld.cu/scielo.php?script=s ci_arttext\&pid=S1561-

31942015000300011

18. Osorio V, Ibarra R del CC. Cuidadores y cuidadoras familiares de personas con enfermedad crónica en Colombia: más similitudes que diferencias. Rev Científica Salud Uninorte. 2015; [citado 2019 Agosto 15] 31. Disponible:

http://rcientificas.uninorte.edu.co/inde x.php/salud/article/viewArticle/6988/8 025

19. Carreño, S. Chaparro L. Calidad de vida de los cuidadores de personas con enfermedad crónica. Aquichan, 2016. [Citado 2019 octubre 21]16 (4), Disponible: http://www.redalyc.org/pdf/741/74148 832004.pdf

20. De Valle M, Hernández I, Zúñiga M y Martínez P. Sobrecarga y Burnout en cuidadores informales del adulto mayor. Enferm Univ. 2015; [citado 2019 octubre 21] 12(1):19-27. Disponible:

http://www.scielo.org.mx/pdf/eu/v12n 1/v12n1a4.pdf

21. Ramón-Arbués, E., Martínez-Abadía, B., \& Martín-Gómez, S. Factores determinantes de la sobrecarga del cuidador. Estudio de las diferencias de género. [internet]. [citado $10 \mathrm{abr}$ de 2020]. Atención Primaria, 49(5), 308. Disponible:

https://www.ncbi.nlm.nih.gov/pmc/arti cles/PMC6876004/

22. Rizo Vázquez, A. C., Molina Luís, M., Milián Melero, N. C., Pagán Mascaró, P. E., \& Machado García, J. Caracterización del cuidador primario 
Olarte Rueda G, Largo Cardenas YC, León Acosta DL, Sánchez Cubides LSDR, Ríos

Gómez LM

de enfermo oncológico en estado avanzado. Revista Cubana de Medicina General Integral, 32(3), 0-0.2016. [internet]. [citado 9 junio de 2020]: http://scielo.sld.cu/scielo.php?script=s ci_arttext\&pid=S0864-

21252016000300005

23. Borda,J., Guayacán,V., Monsalve,M., Arévalo, M. Disminución de sobrecarga en cuidadores de adultos mayores asistentes a Centro Día. Pontif Univ Javeriana. 2016;1. [internet]. [citado 9 junio de 2020].: https://repository.javeriana.edu.co/bitst ream/handle/10554/21357/BordaGonz alezJuanita2016.pdf? sequence $=1 \&$ isA1 lowed=y

24. Navarro-Sandoval C, UriosteguiEspíritu LC, Delgado-Quiñones EG, et al. Depresión y sobrecarga en cuidadores primarios de pacientes geriátricos con dependencia física de la UMF 171. Rev Med Inst Mex Seguro Soc. 2017;55(1):25-31.

25. Rodríguez-Albañil, Y. y QuembaMesa, M. "Nivel de sobrecarga en cuidadores informales de personas en situación de discapacidad cognitiva de Duitama. Estudio exploratorio." Revista Investigación en Salud Universidad de Boyacá 5.1 (2018): 8698.
26. Piñeiro, I., Rodríguez, S., Albite, A., Freire, C. ., \& Ferradás, M. del M. (2017). Sobrecarga y salud percibida en cuidadores informales de pacientes con enfermedad mental. European Journal of Health Research, 3(3), 185-196. https://doi.org/10.30552/ejhr.v3i3.75

27. Romero, Z.,Cuba, S. Síntomas somáticos en cuidadores de pacientes geriátricos con o sin sobrecarga, del área Perú. Rev Med Hered. 2013;2049. Dsiponible en: http://www.scielo.org.pe/pdf/rmh/v24n 3/v24n3ao4.pdf

28. Lemus Fajardo NM, Linares Cánovas LB, Linares Cánovas LP. Nivel de sobrecarga de cuidadores deadultos mayores frágiles. Rev Ciencias Médicas [en línea]. 2018 [consulta: fecha de acceso]; 22(5): 894-905. Disponible en: http://revcmpinar.sld.cu/index.php/pub licaciones/article/view/3636

29. Rodríguez-Albañil Y, Quemba-Mesa MP. Nivel de sobrecarga en cuidadores informales de personas en situación de discapacidad cognitiva de Duitama, estudio exploratorio. Revista Investig Salud Univ Boyacá. 2018;5:(1):86-98. doi:

https://doi.org/10.24267/23897325.270 